\title{
Review of: "Deep learning-based algorithm for lung cancer detection on chest radiographs using the segmentation method"
}

Yu Gu

Potential competing interests: The author(s) declared that no potential competing interests exist.

Thanks for the possibility to review this paper. I also have a couple of comments.

1. The authors said that their CNN architecture was based on the encoder-decoder architecture to output segmentation. Their model should be presented in detail.

2. Ablation experiment was not made to prove the effectiveness of the each part of the model.

3. The hyperparameter experiment should also be added to select optimal configurations.

4. The authors use the encoder-decoder architecture to improves the model robustness to noise and overfitting. However $\square$ The authors use a training dataset and a test dataset. How do the authors judge overfitting as they do not split dataset for validation.

5. Did authors adopt data augmentation to enlarge the positive samples?

6. How did authors select negative samples in training process? Did they adopt hard negative samples mining?

7. It is suggested that authors write the ideas on how to further improve their results in the future work. 